\title{
Tuberculosis on the rise in southern Mozambique (1997-2012)
}

\author{
To the Editor:
}

Tuberculosis (TB) remains an important public health concern and a leading cause of disease and death worldwide. Mozambique is one of the few high-burden countries where incidence rates have not improved in recent years. The estimated TB incidence rate in 2014 was 552 cases per 100000 population and the estimated case detection rate is very low at just around $39 \%[1,2]$. The National Tuberculosis Control Programme (NTP) in Mozambique was launched in 1977 and expanded nationwide in 1985 [3]. The TB patient registration system began in 1984 and short-course therapy followed in the late eighties [3, 4], despite a civil war which resulted in a shortage of qualified medical staff and supplies, and fragile deployment systems. By the year 2000, the Directly Observed Therapy, Short-Course (DOTS) strategy had complete coverage in all districts of Mozambique [4].

Until now, very few studies on the burden of disease in the country have been published and none of them show temporal trends in relevant TB indicators. However, recent studies conducted in southern Mozambique show a high TB burden among children and people living with HIV, including high mortality [5-7]. To address this we evaluated how the TB incidence rates and other key TB indicators evolved from 1997-2012 in a high TB/HIV-burden area of southern Mozambique [6, 8]. This retrospective descriptive study was conducted by the Centro de Investigação em Saúde de Manhiça (CISM; the Manhiça Health Research Center), located in the Manhiça district of southern Mozambique. Manhiça District covers an area of $2380 \mathrm{~km}^{2}$ in the northern portion of Maputo province. According to the 2015 census conducted by CISM it has 178000 inhabitants living in around 39000 households. Other health and demographic characteristics of the population of the district have been described elsewhere [9].

All patients starting TB treatment during the period 1997-2012 were included and data from registry books based on World Health Organization (WHO) international standards, which were used throughout the country, were entered into an electronic database (including treatment outcomes). During the study period, TB patients were only diagnosed and registered in the two main health centers: Manhiça District Hospital $(\mathrm{MDH})$ and Xinavane Rural Hospital. There were also 12 peripheral health-care centers, where treatment was supervised under a modified directly observed treatment (DOT) system once weekly. In our analysis, we retrospectively applied the revised 2013 WHO definitions [10] to all cases. TB cases were classified as 'new', 'previously treated', or 'unknown TB treatment history'. Following WHO definitions for incident cases, we considered in this category all new and relapse cases [10]. Multidrug-resistant tuberculosis (MDR-TB) was registered from 2011. Diagnosis was based on smear microscopy (Ziehl-Neelsen stain) following the International Union Against Tuberculosis and Lung Disease (IUATLD) smear-grading scheme. Culture or Xpert ${ }^{\oplus}$ MTB/RIF testing was not done routinely during the study period. For treatment outcomes, patients were classified as 'cured', 'treatment completed', 'treatment failed', 'died', 'lost to follow up', or 'not evaluated'. Treatment success was defined as the sum of 'cured' and 'treatment completed'.

Our estimation of population at risk for each year between 1997 and 2007 was based on the annual demographic estimates of the Spanish National Institute of Statistics (INE) for Mozambique. From 2008 onwards, we applied CISM's Demographic Surveillance System (DSS) growth rates to the previous population estimates. Our temporal unit of analysis was annual but some figures were reported in 4 year intervals in order to minimise short-period effects. TB/HIV analysis was only possible beginning in 2007, when HIV testing was fully implemented across NTP offices. However, HIV counselling, testing and treatment was provided free of charge to all of the population in the district health centers from 2005.

@ERSpublications

In Manhiça District, Mozambique, tuberculosis incidence rates underwent a 3-fold increase over 16 years (1997-2012) http://ow.ly/mkWG306wvGb

Cite this article as: García-Basteiro AL, Miranda Ribeiro R, Brew J, et al. Tuberculosis on the rise in southern Mozambique (1997-2012). Eur Respir J 2017; 49: 1601683 [https://doi.org/10.1183/ 13993003.01683-2016]. 


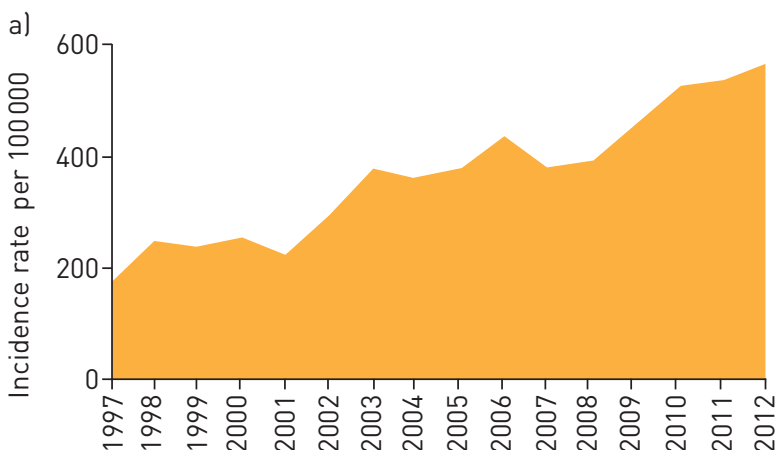

Year

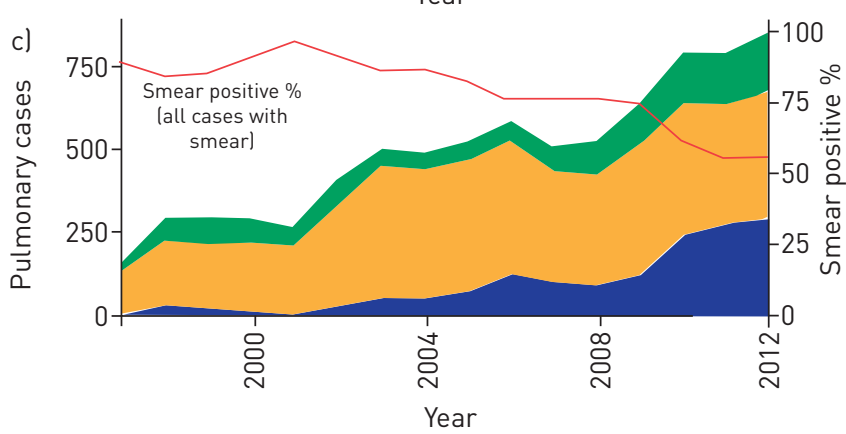

Smear result $\square$ No result $\square$ Positive $\square$ Negative

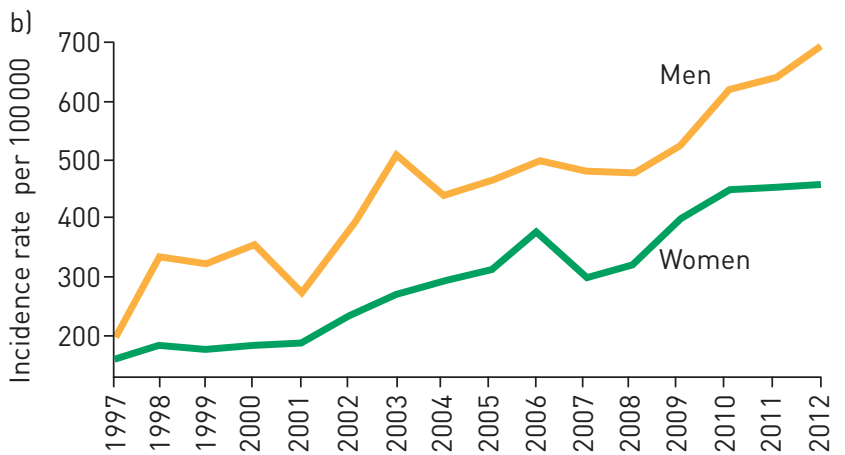

Year

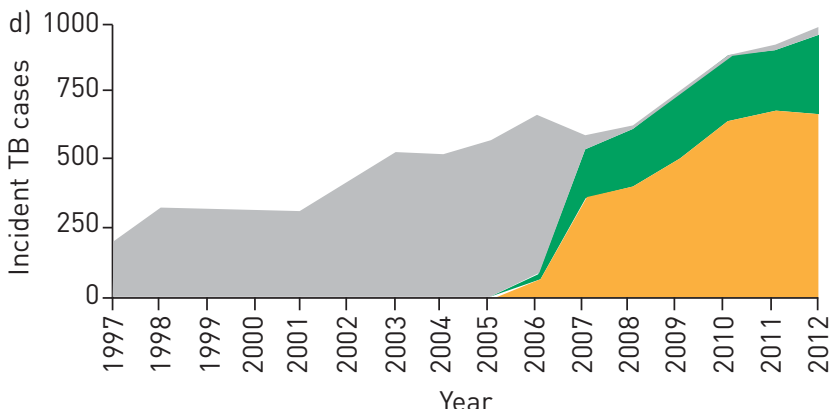

HIV status $\square$ Positive $\square$ Negative $\square$ Unknown

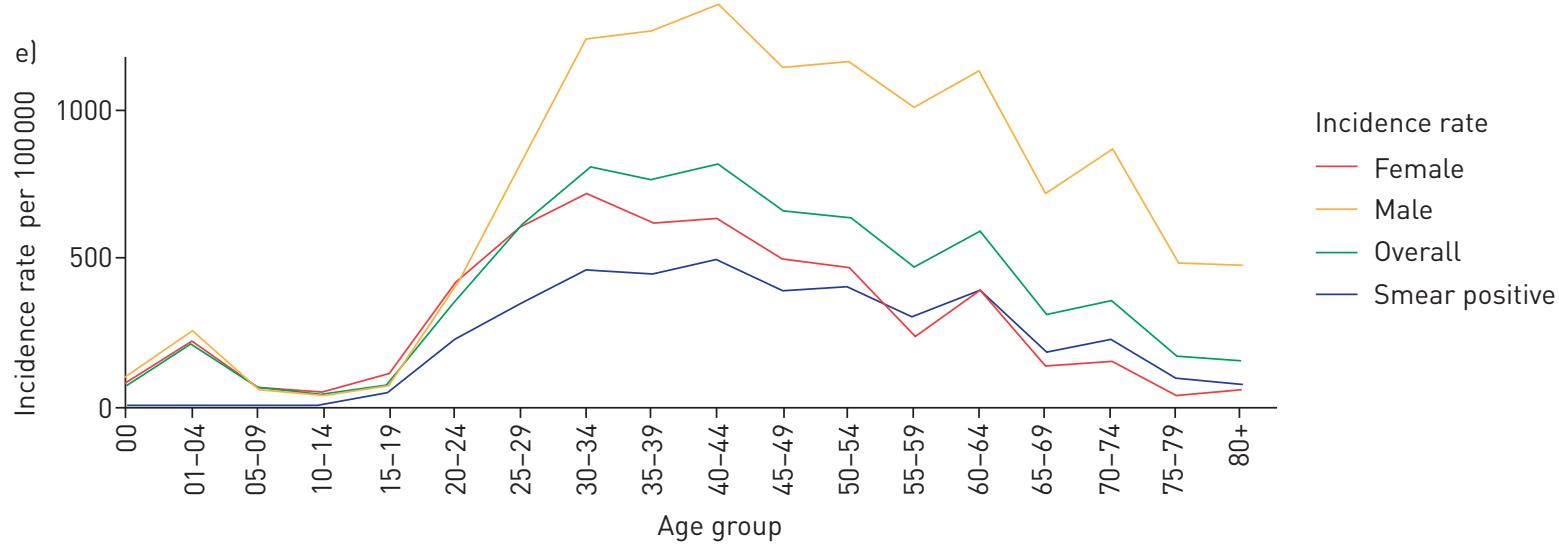

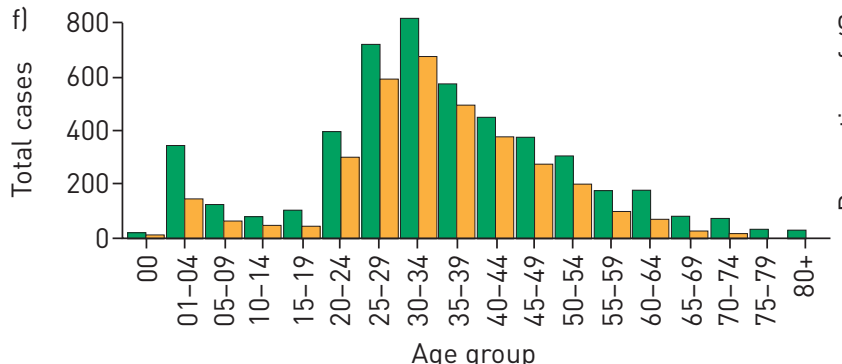

Status
Age group

TB $\square$ TB\&HIV

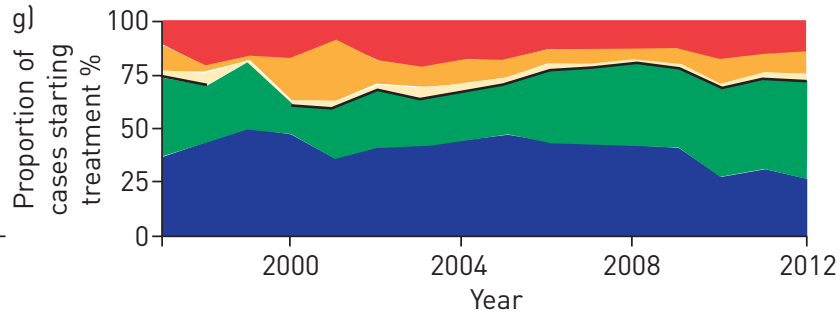

Outcome

Cured

Treatment completed
Treatment failed $\square$ Not evaluated: transferred out

FIGURE 1 a) Overall tuberculosis (TB) incidence rate per 100000 population in the district of Manhiça (1997-2012). b) Sex-specific TB incidence rates per 100000 population. c) Trends in smear testing among pulmonary TB patients. d) Trends in TB case notification labsolute numbers by HIV status). e) TB incidence rates (overall, sex-specific and laboratory confirmed) by age group (1997-2012). f) Number of TB cases by age and HIV status (2007-2012). g) Temporal trends in TB treatment outcomes (1997-2012). 
CISM's scientific and bioethical committees approved this research proposal (CIBS/070/2015) and administrative permission to use official information contained in the NTP registry books was granted by the Manhiça District health officer.

Over the 16-year period of the study, 9575 cases of TB were registered of which 8985 were incident cases. The median age of the subjects was 33 years (interquartile range: $24-45$ ) and $55.9 \%$ of them were men. The TB incidence rate increased nearly three-fold from 1997 to 2012 for all age groups, from an annual incidence rate of 174 cases per 100000 population in 1997 to 573 incident cases per 100000 population in 2012 (figure 1a). It was consistently higher in males than in females among those above 20 years of age and those aged 40-44 years had the highest incidence rate of all age groups (819 per 100000 population annually) (figure $1 \mathrm{~b}$ and figure $1 \mathrm{e}$ ). Around $9.4 \%$ of subjects ( 878 of the 9373 cases with available data on history of TB treatment) were previously treated patients and this proportion did not undergo major variation across the years. Furthermore, extrapulmonary TB (EPTB) accounted for $15.4 \%$ (1477) of all cases.

From 2007 onwards, HIV status was recorded in more than $98 \%$ of registered incident TB patients (figure $1 \mathrm{~d}$ and figure 1f). The total number of incident TB cases with known HIV status was 4731 (52.7\%) and, among these patients, 3324 (70.3\%) were HIV-positive. Since 2007, the proportion of HIV-positive cases has remained relatively constant, hovering at around 70\%. Of the 843 EPTB patients with known HIV status, 641 (76.0\%) were HIV-positive. Smear results were available for $83.0 \%$ of all pulmonary cases and $75.6 \%$ of these were positive (figure 1c). The proportion of smear-positive results decreased during the study period, from $87.2 \%$ in $1997-2000$ to $61.5 \%$ in $2009-2012$. HIV-infection was more frequent among smear-negative cases $(80.2 \%)$ than among smear-positive cases $(67.1 \%)(\mathrm{p}<0.001)$.

The overall proportion of treatment success was $68.2 \%$ (6534) while $15.4 \%$ (1478) of patients died during TB treatment (figure 1g). Women experienced fewer deaths (595 cases; 14.8\%) than men (883; 17.0\%) during treatment $(\mathrm{p}=0.003)$ and mortality rates were relatively constant during the study period. Among those with known HIV status, the mortality rate during TB treatment was much higher in HIV-positive TB patients compared to HIV-negative ones (17.3\% versus $8.3 \%$, respectively; OR $2.21,95 \%$ CI 1.81-2.72).

TB incidence rates have increased remarkably (almost three-fold) in the district of Manhiça from 1997 to 2012. As this has happened in different regions of the world [11] it could be attributable to the HIV epidemic [6], although no seroprevalence data is available at population level to confirm this association. Data on the prevalence of HIV infection in women attending the antenatal clinic showed that HIV was experiencing an upward trend in the district (23.6\% in $2003-2004$ to $29.4 \%$ in 2010$)$ [8, 12]. The increase in the proportion of smear-negative cases would also support this explanation. However, the prevalence of HIV among TB cases has remained stable since systematic testing was implemented by the NTP in 2008 and the TB incidence rates kept rising during the period 2008-2012, suggesting increased awareness and improved case detection by the health system, accompanied by increased access to health services by the population. This latter argument was also supported by the relatively constant male/female ratio and fatality level during the study period, and would also suggest an already high prevalence of HIV back in 1997. Other african countries, such as South Africa, Kenya and Zambia, have experienced declining trends in TB notification since 2007, likely attributable to improved TB and HIV care [13-15]. Nonetheless, it should be noted that the constantly poor treatment outcomes over time in Mozambique may be partially attributable to suboptimal management along the TB care cascade.

The origins of the remarkable differences in TB incidence rate by sex remain unclear. Despite HIV incidence being higher in females in young adulthood [8], the migration of male workers to hotspots of high TB and HIV transmission could play an important role in the observed patterns of disease. Although there is no reliable information on coverage levels of antiretroviral treatment (ART) before 2012, when the HIV/TB one-stop model was implemented in the district (with HIV treatment being provided during TB treatment and monitored by the NTP officers), the rapidly increasing ART coverage reported nationally (29\% in 2011 and $81 \%$ in 2014) [1] should lead to improved mortality rates [15].

Analysing the factors influencing TB and their trends over time will contribute to better understanding of the current TB epidemic in Mozambique and inform public health policy. Our study was based in the programmatic passive surveillance system and notification rates probably underestimate incidence rates. In addition, some diagnosed cases might not have started treatment, might not have been registered and thus might not have been taken into account in this analysis. However, these results enable the study of trends in key indicators with no known major changes in the reporting, diagnostic tools and algorithms occurring during a 16-year period, which is in itself an important concern.

Alberto L. García-Basteiro ${ }^{1,2,3}$, Rafaela Miranda Ribeiro ${ }^{1}$, Joe Brew ${ }^{1,2}$, Charfudin Sacoor ${ }^{1}$, Salomé Valencia ${ }^{1,2}$, Helder Bulo ${ }^{1}$, Frank Cobelens ${ }^{3}$ and Eusebio Macete ${ }^{1}$

${ }^{1}$ Centro de Investigação em Saúde de Manhiça (CISM), Vila da Manhiça, Mozambique. ${ }^{2}$ ISGlobal, Barcelona Ctr. Int. Health Res. (CRESIB), Hospital Clínic - Universitat de Barcelona, Barcelona, Spain. ${ }^{3}$ Dept of Global Health, Academic Medical Centre, Amsterdam Institute for Global Health and Development (AIGHD), Amsterdam, The Netherlands. 
Correspondence: Alberto L García-Basteiro, Rua 12 Cambeve, Manhiça, Maputo 1929, Mozambique.

E-mail: alberto.garcia-basteiro@manhica.net

Received: Aug 242016 | Accepted after revision: Nov 042016

Support statement: Alberto L. García-Basteiro was the beneficiary of a fellowship from the Rio Hortega programme of the Instituto de Salud Carlos III (grant number: CM12/00246). In addition, this work was partially supported by the Erasmus Mundus Joint Doctorate Programme of the European Union through a training grant to Alberto L. García-Basteiro. No specific funding was requested from any institution for the conduct of this study.

Conflict of interest: None declared.

Acknowledgements: The authors would like to thank the District Health Authorities (Fausta Temba) and NTP for their constant support. We would also like to thank Henriquez Mbeve and Alberto Bila, as well as all of the CISM and MDH clinicians and staff who have contributed to TB diagnosis in the district over the study period.

\section{References}

1 World Health Organization. Global Tuberculosis Report 2015 (who/htm/tb/2015.22). Geneva, World Health Organization, 2015. Available from: www.who.int/tb/publications/global_report/gtbr15_main_text.pdf Date last accessed: November, 2015

2 López-Varela E, Augusto OJ, Guerra L, et al. Low paediatric tuberculosis case detection rate in southern Mozambique. Eur Respir J 2016; 47: 1003-1005.

3 Salomao MA. The National Tuberculosis Control Programme in Mozambique, 1985-1990. Bull Int Union Tuberc Lung Dis 1991; 66: 175-178.

4 Cliff J, Walt G, Nhatave I. What's in a name? Policy transfer in Mozambique: DOTS for tuberculosis and syndromic management for sexually transmitted infections. J Public Health Policy. 2004; 25: 38-55.

5 López-Varela E, Augusto OJ, Gondo $\mathrm{K}$, et al. Incidence of tuberculosis among young children in rural Mozambique. Pediatr Infect Dis J 2015; 34: 686-692.

6 García-Basteiro AL, López-Varela E, Respeito D, et al. High tuberculosis burden among people living with HIV in southern Mozambique. Eur Respir J 2015; 45: 547-549.

7 García-Basteiro AL, Respeito D, Augusto OJ, et al. Poor tuberculosis treatment outcomes in Southern Mozambique (2011-2012). BMC Infect Dis 2016; 16: 214.

8 González R, Munguambe K, Aponte J, et al. High HIV prevalence in a southern semi-rural area of Mozambique: a community-based survey. HIV Med 2012; 13: 581-588.

9 Sacoor C, Nhacolo A, Nhalungo D, et al. Profile: Manhiça Health Research Centre (Manhiça HDSS). Int Epidemiol 2013; 42: 1309-1318.

10 World Health Organization. Definitions and reporting framework for tuberculosis - 2013 revision (who/htm/tb/ 2013.2). Geneva, World Health Organization, 2013. Available from: http://apps.who.int/iris/bitstream/10665/ 79199/1/9789241505345_eng.pdf?ua=1 Date last accessed: November, 2016. Date last updated: December 2014.

11 Tran NB, Houben RM, Hoang TQ, et al. HIV and tuberculosis in Ho Chi Minh City, Vietnam, 1997-2002. Emerg Infect Dis 2007; 13: 1463-1469.

12 Menéndez C, Bardají A, Sigauque B, et al. Malaria Prevention with IPTp during pregnancy reduces neonatal mortality. PLoS One 2010; 5: e9438.

13 Yuen CM, Weyenga HO, Kim AA, et al. Comparison of trends in tuberculosis incidence among adults living with HIV and adults without HIV-Kenya, 1998-2012. PLoS One 2014; 9: e99880.

14 Kapata N, Chanda-Kapata P, O'Grady J, et al. Trends of Zambia's tuberculosis burden over the past two decades. Trop Med Int Health 2011; 16: 1404-1409.

15 Nanoo A, Izu A, Ismail NA, et al. Nationwide and regional incidence of microbiologically confirmed pulmonary tuberculosis in South Africa, 2004-12: a time series analysis. Lancet Infect Dis 2015; 15: 1066-1076. 